\title{
O Efeito da Interatividade E do Suporte Técnico na InTENÇÃo de Uso de UM Sistema de E-Learning
}

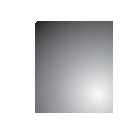

\author{
The Effect of Interactivity and Technical Support in the use \\ Intention of an E-learning System
}

\begin{abstract}
Angilberto Sabino de Freitas
Doutor em Administração pela PUC-Rio. Pesquisador e Professor adjunto do Programa de Pós-Graduação em Administração da Universidade do Grande Rio. Rio de Janeiro, RJ. Brasil.E-mail: angilberto.freitas@gmail.com
\end{abstract}

Jorge Brantes Ferreira

Doutor em Administração pelo COPPEAD. Pesquisador e Professor do Programa de Pós-Graduação em Administração da PUC Rio. Rio de Janeiro, RJ. Brasil. E-mail: jorgebf@gmail.com

Rodrigo Antônio Garcia

Mestre em administração pela Universidade do Grande Rio. Rio de Janeiro, RJ. Brasil. E-mail: prof.rodrigogarcia@gmail.com

Renata Kurtz

Doutora em Administração pela PUC-Rio. Professora e Pesquisadora em Administração da UERJ. Rio de Janeiro, RJ. Brasil.

E-mail: renatakurtz@gmail.com

\section{Resumo}

Este trabalho tem como objetivo identificar as variáveis que influenciam o uso do e-learning por alunos de um curso de graduação a distância de uma Instituição de Ensino Superior (IES). Foi identificado que o Modelo de Aceitação de Tecnologia (Technology Acceptance Model - TAM), de Davis (1989), com a introdução das variáveis externas interatividade e suporte técnico seria o mais adequado para avaliar a intenção do aluno em seguir usando o e-learning. Foi aplicado um questionário pela internet que resultou em uma amostra de 260 alunos. Os dados obtidos foram analisados por meio da Modelagem de Equações Estruturais (SEM) $e$ os resultados confirmaram a validade de todas as hipóteses, confirmando a importância da interatividade e do suporte técnico para os alunos continuarem a usar o e-learning.

Palavras-chave: E-learning. Modelo de Aceitação de Tecnologia. Interatividade. Suporte Técnico.

\section{Abstract}

The objective of this research is to identify the variables that influence the use of e-learning by students in an undergraduate distance course of a Higher Education Institution (HEI). It was identified that Davis' (1989) Technology Acceptance Model (TAM) with the introduction of interactivity and technical support as external variables would be the most appropriate to assess the intention of the student to follow using e-learning. A questionnaire was sent over the internet and resulted in a sample of 260 students. The data were analyzed by Structural Equation Modeling (SEM) and the results confirmed the validity of all the hypotheses confirming the importance of interactivity and technical support for the students to continue to use e-learning.

Keywords E-learning. Technology Acceptance Model. Interactivity. Technical Support. 


\section{INTRODUÇÃo}

A partir da década de 1990, com o surgimento e a expansão da Internet, grandes alterações ocorreram no acesso à informação $e$ na maneira das pessoas se comunicarem. Essas mudanças impactaram o processo de ensino-aprendizagem de tal forma que a $\mathrm{EaD}$ (Educação a distância) passou a destacar-se como uma das mais importantes ferramentas de difusão de conhecimento e educação (MAIA, 2003). Segundo Freitas (2009), o uso do computador como mediador das interações no ambiente de aprendizagem, representa a $3^{\mathrm{a}}$ geração na evolução tecnológica da $\mathrm{EaD}$, chamada de e-learning. Assim, o e-learning pode ser entendido como o uso de tecnologias digitais no processo de ensino-aprendizagem, com a Internet como suporte, podendo ser presencial ou a distância.

$\mathrm{O}$ aumento na utilização do e-learning como ferramenta do processo de ensino-aprendizado, sobretudo por Instituições de Ensino Superior (IES), é uma tendência que vem sendo amplamente observada no contexto nacional. De acordo com o Censo EaD.br de 2014, o número de cursos EaD totalmente a distância regulamentados é de 1.772. Destes, 505 são de graduação e 489 são da área de Ciências Sociais. Os cursos se dividem por todos os níveis: Educação de Jovens e Adultos (EJA) (Fundamental e Médio), Técnico profissionalizante e Superior (Sequencial, Graduação e Pós-graduação). O que se observa, é que a maior concentração de alunos em cursos a distância, cerca de $64,7 \%$ dos alunos matriculados, ocorre em cursos de Graduação (ABED, 2014).

Apesar desses números, verifica-se um elevado grau de evasão nessa modalidade de ensino (PACHECO; NAKAYAMA; RISSI, 2015). Isso leva as instituições afligidas a buscarem uma forma de minimizar o problema e os impactos financeiros causados por ele. Segundo Favero e Franco (2006), o índice de evasão da EaD é superior ao do ensino presencial, chegando a $30 \%$ em cursos totalmente à distância. Já o relatório da Associação Brasileira de Educação a Distância (ABED) reporta uma média de evasão nos cursos a distância das instituições pesquisadas da ordem de $25 \%$, mas aponta instituições com índices de até $75 \%$ (ABED, 2014).

É possível que esses altos índices estejam relacionados ao modelo de educação a distância adotado e a sua plataforma de ensino, chamada de Ambiente Virtual de Aprendizagem (AVA). De acordo com Favero e Franco (2006), uma das questóes levantadas que poderia levar o aluno a abandonar o curso antes de seu termino estaria ligada ao modelo de e-learning adotado pela instituição. Segundo os autores, as instituições que adotam modelos que usam AVAs que permitem uma maior interação entre alunos e entre alunos e professores tendem a ter um índice de evasão menor em relação àquelas em que há menor interação entre os participantes do curso. Dessa forma, a questão da interatividade se torna um importante fator a ser investigado.

Já em relação ao suporte que é oferecido pela organização tanto administrativo quanto técnico, Abbad, Zerbini e Souza (2010) identificaram que na falta deles os participantes de cursos a distância em educação corporativa estariam mais propensos e abandonar o curso. Pode-se, então, inferir que no contexto das IES não seria diferente, ou seja, a falta de suporte técnico poderia ser um fator a levar o aluno a desistir do curso.

Apesar de existirem outros fatores que procuram explicar a intenção de uso do e-learning, em casos em que o aluno já iniciou o curso, esse dois fatores parecem ser os mais propensos a influenciar o aluno a abandonar ou não o curso. Assim, investigar a influência da interatividade e do suporte técnico no comportamento do estudante no sentido de mantê-lo matriculado até o final do curso se torna uma ação importante para compreender a sua descontinuação do uso do e-learning. Tal compreensão poderia levar os gestores a adotar medidas para reduzir a evasão de alunos. Portanto, o objetivo deste estudo é propor um modelo para verificar como as variáveis aqui apontadas influenciam a intenção do aluno de cursos de graduação a distância de uma instituição de ensino superior de continuar usando o e-learning como uma ferramenta no processo de ensino-aprendizagem.

\section{ReVisão de Literatura}

Esta seção tem por objetivo descrever o arcabouço teórico que da suporte a esta pesquisa. Inicialmente é apresentado modelo para avaliar a aceitação de tecnologia. Na seção seguinte serão apresentados os fatores que influenciam o uso de sistemas de e-learning 
e, por fim, um modelo e suas hipóteses para verificar a intenção de uso por alunos de um sistema de e-learning.

\subsection{O Modelo Technology Acceptance Model (TAM)}

O Modelo de Aceitação de Tecnologia (Technology Acceptance Model - TAM), apresentado por Davis, Bagozzi e Warshaw (1989), tem sido amplamente utilizado em estudos sobre aceitação e uso das tecnologias computacionais e sistemas de informação (YOUSAFZA; FOXALL; PALLISTER 2007), como é o caso de um sistema de e-learning. De acordo com Davis (1989), os indivíduos tendem a usar uma determinada tecnologia quando percebem que a mesma irá melhorar seu desempenho. O modelo TAM, então, avalia o comportamento dos usuários por meio de dois fatores cognitivos: (1) utilidade percebida e (2) facilidade de uso percebida. Entretanto, apesar de o usuário compreender a utilidade da tecnologia (utilidade percebida), sua adoção poderá ser prejudicada em função do seu grau de complexidade (facilidade de uso percebida) (DAVIS; BAGOZZI; WARSHAW, 1989).

A utilidade percebida refere-se ao grau de crença do indivíduo em que a utilização de um determinado sistema poderá melhorar o seu desempenho. Está relacionada à percepção de vantagem a ser obtida ao adotar determinado sistema. Já a facilidade percebida refere-se ao grau de crença do indivíduo em que a utilização de determinado sistema poderá diminuir seu esforço. Ou seja, está relacionada à percepção de redução de esforço (DAVIS, 1989).

Os outros construtos do modelo são a atitude, a intenção comportamental de uso e, eventualmente, pode-se medir o uso real do sistema. A atitude se relaciona à avaliação do indivíduo sobre seu comportamento de uso de determinado sistema, exercendo influência direta sobre a intenção comportamental de uso e mediando o efeito da utilidade percebida e da facilidade de uso percebida na intenção comportamental de uso. A intenção comportamental de uso, por sua vez, está relacionada ao grau de intenção do indivíduo em se comportar de determinada forma.

Davis, Bagozzi e Warshaw (1989) testaram e validaram o modelo TAM, propondo também a possibilidade de inserção de variáveis externas que pudessem influenciar na aceitação de uma tecnologia, aumentando assim o poder explanatório do modelo (Figura 1).

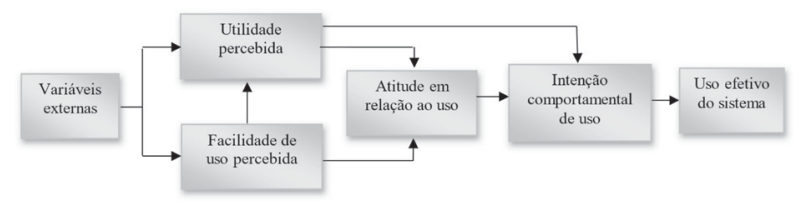

Figura 1: Modelo TAM com variáveis externas Fonte: Davis, Bagozzi e Warshaw (1989)

Para Dishaw e Strong (1999), o modelo TAM fornece uma base teórica muito útil para explorar os fatores que explicam a utilização de um software e sua ligação com o desempenho do usuário. Apesar da existência de outros modelos para medir a aceitação de tecnologias computacionais, como o Modelo do Processo de Difusão de Tecnologia da Informação (STRAUB, 1994), a Teoria do Comportamento Planejado (Theory of Planned Behaviour - TPB) de Ajzen (1991), o TAM2 (VENKATESH; DAVIS, 2000), a Teoria Unificada de Aceitação e Uso de Tecnologia (Unified Theory of Acceptance and Use of Technology - UTAUT) de Venkatesh, (2003), que é uma tentativa de unificar diversos modelos anteriores $e$ a versão revisitada do UTAUT, o UTAUT 2 (VENKATESH; THONG; XU, 2012), o TAM tem sido o mais utilizado em pesquisas devido a sua parcimônia a alto poder explanatório, além de permitir a inclusão de variáveis externas particulares de cada contexto investigado (LEE; KOZAR; LARSEN, 2003; MERCHANT, 2007).

\subsection{Fatores que Influenciam o Uso de Sistemas de E-learning}

Segundo Park (2009), diversas Instituições de Ensino enfrentam dificuldades na implementação do e-learning pelo fato de tentarem replicar a experiência adquirida no ensino presencial, não atentando para as especificidades desta modalidade, principalmente quanto aos fatores críticos de aceitação de sistemas de informação por parte dos alunos. Pesquisas tem mostrado que experiências em implementar o e-learning fracassam por causa da não compreensão de fatores que facilitariam a sua adoção (FREITAS; BANDEIRA-DE-MELLO, 2012).

Apesar de os sistemas de e-learning serem particularmente complexos (FREITAS; BANDEIRA-DE-MELLO, 2012), Arbaugh (2005) argumenta que, 
dentro do contexto educacional, o modelo TAM tem sido amplamente utilizado para explicar a aceitação de novas tecnologias de informação por parte dos usuários na área de gestão da educação, principalmente por permitir a inclusão de novas variáveis que afetariam a utilidade percebida e a facilidade de uso percebida.

Variáveis externas como experiência em uso de computadores (PAPASTERGIOU; SOLOMONIDOU, 2005), utilidade do conteúdo (ONG; LAI, 2006), conteúdo de internet (ALSHARE; ALKHATEEB, 2008), normas subjetivas (VAN RAAIJ; SCHEPERS, 2008; PARK, 2009), autoeficácia computacional (ONG; LAI, 2006; CHANG; TUNG, 2008; PARK, 2009), suporte técnico (NGAI; POON; CHAN, 2007), percepção de prazer (ROCA; GAGNÉ, 2006), capacidade de inovação pessoal $e$ ansiedade (VAN RAAIJ; SCHEPERS, 2008), características do sistema (CHANG; TUNG, 2008; PARK, 2009), fatores motivacionais (PARK; LEE; CHEONG, 2007), compatibilidade (CHANG; TUNG, 2008) têm sido testadas no modelo com o objetivo se encontrar uma melhor compreensão de como o indivíduo tende a adotar um sistema de e-learning

Uma vez já usuário de um sistema de e-learning, o aluno pode ao longo do curso se sentir desmotivado por diversas razões e abandonar o curso. A sua não intenção em seguir pode ser reflexo de uma série de fatores associados à estrutura do e-learning que podem ser determinantes para abandonar o uso do sistema e consequentemente abandonar o curso. Dois desses fatores identificados na literatura dizem respeito à interatividade e ao suporte técnico.

Em uma pesquisa exploratória, Favero e Franco (2006) apontaram que Instituições de ensino que adotam sistemas de e-learning que permitem uma maior interação entre alunos e entre alunos e professores tendem a ter um índice de evasão menor em relação àquelas em que há menor interatividade entre os participantes do curso. Assim, pode-se supor que a questão da interatividade pode ser um importante fator para que o estudante continue usando o e-learning. Já Abbad, Zerbini e Souza (2010) identificaram que a falta de suporte técnico nos sistemas de e-learning em cursos corporativos seria uma das causas que levariam os alunos a abandonar o curso. Por sua vez, Ngai, Poon, e Chan (2007) investigaram a influência do suporte técnico e concluíram que esse é um dos principais fatores que leva um indivíduo a aceitar um sistema se e-learning.

Diante do exposto, a seguir é proposto um modelo para avaliar a intenção do aluno em continuar a usar um sistema de e-learning, levando-se em conta dois fatores chaves que afetariam a sua decisão de continuar ou não a usar o sistema: (1) a interatividade e (2) o suporte técnico disponível.

\subsection{Um Modelo para Avaliar a Intenção de Uso de um Sistema de e-learning}

\section{a) Interatividade}

Para Pituch e Lee (2006) um dos elementos-chave do processo de aprendizagem são as interações entre os próprios alunos, as interações entre professores e alunos, bem como a colaboração na aprendizagem que resulta dessas interações.

Um dos principais aspectos das novas plataformas na qual o e-learning tem se baseado é o uso de tecnologias que buscam promover maior interação entre alunos e entre esses e professores e conteúdo. Interações podem ser síncronas ou assíncronas. Assim, a é esperado que a interatividade do sistema seja um dos fatores que podem afetar a adoção de sistemas de e-learning pelos estudantes.

Por Interatividade entende-se a velocidade das respostas dadas, o relacionamento com a turma, o apoio à realização das atividades e o estímulo ao diálogo e à reflexão crítica (PITUCH; LEE, 2006). Em um ambiente interativo, pode-se argumentar que o aluno perceberia uma maior utilidade no uso sistema de $e$ -learning, percebendo assim, uma maior utilidade do mesmo. Assim, é proposta a seguinte hipótese:

$\mathbf{H}_{\mathbf{1}}$ : A Interatividade influencia diretamente, de forma positiva, a Utilidade Percebida.

\section{b) Suporte técnico}

Martins e Kellermanns (2004) observam em sistemas complexos como o e-learning que a disponibilidade de suporte técnico necessário para operar uma nova tecnologia pode reduzir as preocupações do usuário inerentes à sua adoção e uso, achando-o mais fácil de usar. Desse modo, a disponibilidade de suporte técnico para ajudar os usuários na utilização 
do novo sistema pode afetar a percepção de facilidade de utilização do sistema. No sentido contrário, particularmente em contexto de uso de tecnologia para apoio a educação, a carência de um suporte técnico ao usuário de uma determinada tecnologia pode influenciar negativamente sua percepção de facilidade ao usar o sistema, inibindo, assim, a aceitação do mesmo. Sob esse argumento é proposta a segunda hipótese:

$\mathbf{H}_{2}$ : A Disponibilidade de Suporte Técnico influencia diretamente, de forma positiva, a Facilidade de Uso Percebida.

Portanto, para esse estudo, o modelo TAM usou como variáveis externas a influência da interatividade na utilidade percebida e a influência da disponibilidade de suporte na facilidade de uso percebida. As demais relações presentes no modelo TAM permaneceram inalteradas e compuseram as demais hipóteses do modelo, segundo os argumentos a seguir.

Saadé e Bahli (2005) sustentam a relação positiva e direta entre facilidade de uso percebida e utilidade percebida. Para esses autores, estudantes que percebem a facilidade do uso do sistema de e-learning estão mais propensos a considerá-lo mais útil, pois a redução do esforço cognitivo dispendido permite a concentração em outras questões de aprendizagem. Assim, tem-se a terceira hipótese:

$\mathbf{H}_{3}$ : A Facilidade de Uso Percebida influencia diretamente, de forma positiva, a Utilidade Percebida.

Da mesma forma, Davis e Wong (2007), Hung e Cho (2008) e Liu et al (2010), em pesquisas também voltados para e-learning, confirmam a influência direta da utilidade percebida e da facilidade de uso percebida sobre a intenção de uso. Já Martins e Kellermanns (2004), Park (2009), Davis e Wong (2007), Hung e Cho (2008) e Liu et al. (2010) corroboram os construtos utilidade percebida e facilidade de uso percebida como preditores da atitude em relação ao uso. Assim tem-se as hipóteses 4 e 5 :

$\mathbf{H}_{\mathbf{4}}$ : A Utilidade Percebida influencia diretamente, de forma positiva, a Atitude em Relação ao Uso.
$\mathbf{H}_{\mathbf{5}}$ : A Facilidade de Uso Percebida influencia diretamente de, forma positiva, a Atitude em Relação ao Uso.

Martins e Kellermanns (2004) e Park (2009), ao analisar a intenção de uso em relação à aceitação de sistemas de e-learning, indicam que a atitude em relação ao uso, além de influenciar diretamente a intenção comportamental de uso, também atua como mediadora dos efeitos da utilidade percebida e da facilidade de uso percebida na intenção de uso. Finalmente, Martins e Kellermanns (2004) e Park (2009) confirmam a influência da atitude em relação à intenção comportamental de uso. Assim, é proposta a última hipótese do modelo

$\mathbf{H}_{\mathbf{6}}$ : A Atitude em Relação ao Uso influencia diretamemente de, forma positiva, a Intenção de Uso.

Após apresentada as hipóteses, a Figura 2 apresenta a configuração do modelo proposto, descrevendo as relações entre os construtos.

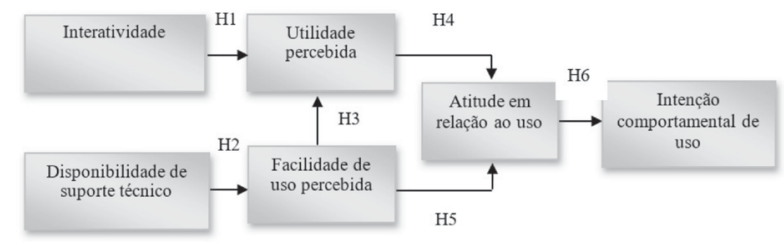

Figura 2: Modelo proposto

Fonte: Adaptada de Davis et al. (1989)

\section{Metodologia}

Com o objetivo de realizar o teste das hipóteses propostas para o estudo, foi realizado um cross-sectional survey (PARASURAMAN; GREWAL; KRISHNAN, 2006) com uma amostra não probabilística da população de interesse. Grande parte dos estudos sobre aceitação de tecnologia, como o e-learning, faz uso desta mesma forma de pesquisa (ONG; LAI, 2006; PARK, 2009), aplicando questionários estruturados a estudantes em um único momento no tempo.

\subsection{Operacionalização das Variáveis}

O modelo proposto é composto de seis hipóteses que buscam explicar a aceitação do e-learning pelos alunos e o relacionamento entre os construtos. Para medição dos construtos envolvidos no modelo proposto 
foram utilizadas escalas já elaboradas e testadas pela literatura. Dessa forma, as escalas utilizadas como base para realização deste estudo são descritas no Quadro 1:

\begin{tabular}{|l|l|}
\multicolumn{1}{|c|}{ Construtos } & \multicolumn{1}{c|}{ Escalas } \\
\hline Intenção de Uso & $\begin{array}{l}\text { Escala de Venkatesh et al. } \\
\text { (2003) composta de 02 itens }\end{array}$ \\
\hline $\begin{array}{l}\text { Atitude em } \\
\text { Relação ao Uso }\end{array}$ & $\begin{array}{l}\text { Escala de Venkatesh e Davis } \\
\text { (2000) composta de 4 itens }\end{array}$ \\
\hline Utilidade Percebida & $\begin{array}{l}\text { Escala de Davis (1989) } \\
\text { composta de 6 itens }\end{array}$ \\
\hline $\begin{array}{l}\text { Facilidade de } \\
\text { Uso Percebida }\end{array}$ & $\begin{array}{l}\text { Escala de Davis (1989) } \\
\text { composta de 6 itens }\end{array}$ \\
\hline $\begin{array}{l}\text { Disponibilidade de } \\
\text { Suporte Técnico }\end{array}$ & $\begin{array}{l}\text { Escala de Martins e Kellermanns } \\
\text { (2004) composta de 03 itens }\end{array}$ \\
\hline Interatividade & $\begin{array}{l}\text { Escala de Pituch e Lee (2006), } \\
\text { composta de 3 itens }\end{array}$ \\
\hline
\end{tabular}

Quadro 1: Construtos e Escalas Utilizadas na Pesquisa Fonte: Elaborado pelos autores deste artigo

As escalas originais foram traduzidas e adaptadas para o português. O instrumento de pesquisa resultou em 24 itens sobre os construtos, em escala Likert de cinco pontos, acrescidas de questões a respeito das variáveis demográficas. Foi realizado um pré-teste com uma pequena amostra da população de interesse. $\mathrm{O}$ objetivo foi avaliar a compreensão dos respondentes sobre o questionário apresentado. Após o pré-teste, pequenos ajustes foram feitos. O resultado final indicou que o questionário estava de fácil compreensão para os respondentes, uma vez que não apresentaram dúvidas em relação às questões.

\subsection{Amostra e Coleta de Dados}

Para a coleta de dados foram aplicados questionários estruturados, enviados eletronicamente a alunos matriculados no curso a distância de graduação em Administração, de uma Instituição de Ensino Superior (IES) do Rio de Janeiro. Essa IES é reconhecida pela sua excelência nas modalidades presencial e à distância, possui cinco cursos de graduação tecnológica, quais sejam: Gestão Comercial, Gestão Financeira, Gestão Pública, Marketing e Processos Gerenciais. Esses cursos são avaliados pelo MEC com nota 5, a nota máxima.
Os alunos são de diversas partes do Brasil e fora do Brasil. Foram enviados 1.302 questionários e obtida uma amostra com 260 respondentes. Não houve dados ausentes e a amostra final foi composta por 260 questionários válidos.

\subsection{Análise dos Dados}

Os dados obtidos foram analisados por meio da técnica de Modelagem de Equações Estruturais (Structural Equation Model - SEM), escolhida por oferecer ao pesquisador a possibilidade de investigar quão bem as variáveis preditoras (variáveis independentes) explicam a variável dependente e, também, qual a variável preditora é a mais importante para o fenômeno estudado (SILVA, 2006) e foram tratados e analisados com o Excel e SPSS (versão 20). Já para as análises estatísticas e multivariadas, foram utilizados os softwares SPSS (versão 20) e AMOS (versão 20).

Segundo Hair et al. (2009), para condução da SEM deve-se primeiro avaliar em um primeiro momento um modelo de mensuração e em seguida um modelo estrutural.

A construção do modelo de mensuração tem o objetivo de especificar os construtos a serem mensurados e suas variáveis relacionadas, enquanto o modelo estrutural permite a representação do diagrama de caminhos no conjunto de equações estruturais, que representam as relações entre variáveis e construtos, definidas a partir do modelo de mensuração.

O modelo de mensuração define as relações entre as variáveis observadas e os construtos latentes não observados, permitindo a avaliação de quanto cada item medido se relaciona com cada fator em particular. O pesquisador, por meio da análise, confirmará se a associação determinada por ele, entre variáveis e construtos, possui de fato confiabilidade (HAIR et al., 2009).

\section{Resultados}

Esta seção tem por objetivo apresentar os resultados, primeiramente descrevendo os resultados do modelo de mensuração e em seguido os resultados do modelo estrutural, em que se apresenta o teste das hipóteses propostas. 


\subsection{Modelo de Mensuração}

Uma análise fatorial confirmatória (CFA) foi realizada para testar a validade, unidimensionalidade e confiabilidade das escalas utilizadas no modelo de mensuração deste estudo. Foram utilizados para avaliar o modelo de mensuração proposto diversos índices de ajuste tanto incrementais quanto absolutos (HU; BENTLER, 1999). Após análise da matriz de covariância dos resíduos padronizados da CFA, foi identificado que alguns itens contribuíam para o ajuste fraco do modelo. Dessa forma, o modelo foi ajustado e refinado com a eliminação de itens de algumas escalas, no intuito de melhorá-lo. A Tabela 1 apresenta os índices de ajuste do modelo de mensuração inicial e após ajustes.

Tabela 1: Índices de Ajuste do Modelo de Mensuração

\begin{tabular}{|c|c|c|}
\hline $\begin{array}{c}\text { ÍNDICE de } \\
\text { AJuste }\end{array}$ & $\begin{array}{c}\text { Modelo } \\
\text { MENSURAÇão - INICIAL }\end{array}$ & $\begin{array}{c}\text { Modelo } \\
\text { MENSURAÇÃo APós } \\
\text { AJuSTES }\end{array}$ \\
\hline$\chi$ 2/d.f. & 3,10 & 3,10 \\
\hline CFI & 0,89 & 0,90 \\
\hline TLI & 0,87 & 0,88 \\
\hline IFI & 0,88 & 0,90 \\
\hline RMSEA & 0,09 & 0,09 \\
\hline SRMR & 0,06 & 0,05 \\
\hline
\end{tabular}

Fonte: Elaborado pelos autores deste artigo

De acordo com esses índices, considera-se o modelo adequado, apesar de não atingir todos os valores exatos sugeridos pela literatura. Os valores obtidos assumem uma aceitação periférica, ou seja, estão bem próximos aos definidos como ideais, permitindo assim, a validação do modelo (HU; BENTLER, 1999; HAIR et al., 2009).

\subsection{Modelo Estrutural}

O modelo estrutural foi analisado após as modificações propostas para o modelo de mensuração inicial, utilizando os indicadores e construtos presentes nesse. O modelo proposto não teve alteração em sua estrutura, apenas foram feitos ajustes em suas escalas, no intuito de se obter um modelo mais robusto. O modelo estrutural obedeceu ao mesmo critério de análise do modelo de mensuração como pode ser visualizado na Tabela 2.

Tabela 2: Índices de Ajuste Modelo Estrutural

\begin{tabular}{|c|c|c|}
\hline $\begin{array}{c}\text { ÍNDICE DE } \\
\text { AJuSTE }\end{array}$ & $\begin{array}{c}\text { Modelo } \\
\text { EstRUtURAL }\end{array}$ & $\begin{array}{l}\text { VAlor Sugerido } \\
\text { PELA Literatura }\end{array}$ \\
\hline$\chi \mathbf{2} /$ d.f. & 3,37 & $\leq 3$ \\
\hline CFI & 0,89 & $\geq 0,90$ \\
\hline TLI & 0,87 & $\geq 0,90$ \\
\hline IFI & 0,89 & $\geq 0,90$ \\
\hline RMSEA & 0,09 & $\leq 0,08$ \\
\hline SRMR & 0,170 & $\leq 0,08$ \\
\hline
\end{tabular}

Fonte: Elaborada pelos autores deste artigo

De acordo com a literatura, os índices apresentados para o modelo possuem aceitação periférica permitindo que as hipóteses do modelo possam ser verificadas (HU; BENTLER, 1999). Com relação à verificação das hipóteses, os resultados do teste das hipóteses apresentaram um suporte empírico para todas as hipóteses formuladas. Todas as relações foram significativas a um nível de significância de 0,001. A Tabela 3 apresenta os coeficientes padronizados encontrados para cada uma das hipóteses propostas nesta pesquisa.

Tabela 3: Teste das Hipóteses

\begin{tabular}{|c|c|c|c|}
\hline Relação Proposta & $\begin{array}{c}\text { Coeficiente } \\
\text { Padronizado }\end{array}$ & P-VALUE & $\begin{array}{l}\text { HiPótese } \\
\text { VERIFICADA }\end{array}$ \\
\hline $\begin{aligned} \mathbf{H}_{1} & \text { : Interatividade } \\
& =>\text { Utilidade }\end{aligned}$ & 0,61 & $<0,001$ & Sim \\
\hline $\begin{array}{l}\mathrm{H}_{2}: \text { Suporte }=> \\
\text { Facilidade de Uso }\end{array}$ & 0,35 & $<0,001$ & Sim \\
\hline $\begin{array}{l}\mathrm{H}_{3} \text { : Facilidade } \\
=>\text { Utilidade }\end{array}$ & 0,42 & $<0,001$ & Sim \\
\hline $\begin{array}{c}\mathrm{H}_{4}: \text { Utilidade } \\
=>\text { Atitude }\end{array}$ & 0,58 & $<0,001$ & Sim \\
\hline $\begin{array}{c}\mathrm{H}_{5}: \text { Facilidade } \\
\quad=>\text { Atitude }\end{array}$ & 0,03 & $<0,001$ & Sim \\
\hline $\begin{array}{l}\mathrm{H}_{6}: \text { Atitude }=> \\
\text { Intenção de Uso }\end{array}$ & 0,72 & $<0,001$ & Sim \\
\hline
\end{tabular}

Fonte: Elaborada pelos autores deste artigo

Pode-se observar que os resultados obtidos revelam que o modelo proposto apresentou bom ajuste e 
conseguiu fornecer evidências da relação entre as variáveis externas - Disponibilidade de Suporte Técnico e Interatividade - com a Intenção de Uso de sistemas de e-learning sob o ponto de vista dos usuários, no caso os alunos. A Figura 3 reapresenta o modelo com os coeficientes padronizados para cada relação (relembrado que todos os coeficientes foram significantes a $<0,001$ ).

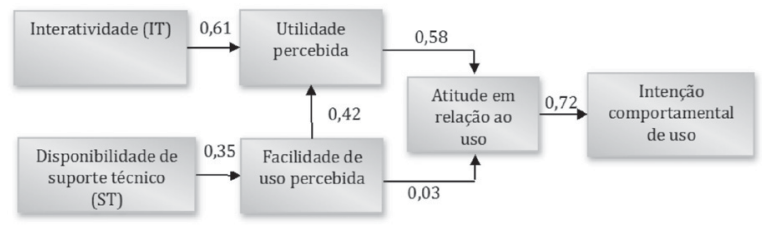

Figura 3: Modelo proposto com coeficientes padronizados Fonte: Adaptada de Davis et al. (1989).

\section{Discussão dos Resultados}

Tal como dito por Martins e Kellermanns (2004) e Park (2009), os resultados obtidos e analisados confirmam a hipótese final deste estudo (H6), em que a análise da intenção de uso, ou seja, a continuação de uso do sistema por alunos de um curso a distância e a aceitação de sistemas de e-learning, demonstra que a atitude em relação ao uso influencia diretamente a intenção comportamental de uso. Percebe-se que a atitude, como uma referência à disposição pessoal do indivíduo relativa a um comportamento específico, relaciona-se com a intenção de um comportamento positivo na adoção de sistemas de Tecnologia da Informação, mais especificamente de sistemas de e-learning, sendo o coeficiente mais forte do modelo $(0,72)$.

Através dos resultados alcançados na presente pesquisa pode-se inferir que os resultados aqui obtidos se alinham a estudos semelhantes em relação às relações testadas (MARTINS; KELLERMANNS, 2004; PARK, 2009; DAVIS; WONG, 2007; HUNG; CHO, 2008; LIU et al., 2010), que apontam que a percepção de utilidade e percepção da facilidade de uso provocam uma influencia positiva e direta na atitude em relação ao uso (H4 e $\mathrm{H} 5$ respectivamente).

Confirmando os estudos de Martins e Kellermanns (2004), Park (2009), Davis e Wong (2007), Hung e Cho (2008) e Liu et al. (2010), os resultados obtidos também indicam que existe influência, direta e positiva de grande magnitude $(0,58)$, da utilidade percebida pelos alunos usuários de sistemas de e-learning sobre sua atitude relativa ao uso destes sistemas de ensino (H4). Por outro lado, a facilidade em relação à atitude, apesar de significativa, provou ter uma influência limitada no modelo, com apenas 0,03. Uma possível explicação pode ser pelo fato de que os alunos hoje já estejam bastante familiarizados com o uso de computadores, o que de certa maneira indica que já não acham difícil o uso de sistemas de e-learning.

Com relação à $\mathrm{H} 3$, a facilidade de uso percebida influenciando diretamente e de forma positiva a utilidade percebida, conforme revelam os testes realizados neste estudo, essa relação se mostrou de uma magnitude mediana $(0,42)$, mas confirmou resultados de trabalhos anteriores (DAVIS; BAGOZZI; WARSHAW, 1989; SAADÉ; BAHLI, 2005).

Com relação a introdução das variáveis externas interatividade e suporte técnico no modelo para avaliar o uso do mesmo por alunos já usuário do e-learning, isso se mostra uma novidade neste tipo de estudo.

No que diz respeito à presença de suporte técnico, recordando Davis (1989), a facilidade percebida diz respeito ao grau de crença do sujeito em que, ao utilizar um certo sistema, poderá ver reduzido seu esforço. Ainda de acordo com Davis (1989), o construto atitude em relação ao uso está conectado a um sentimento diante de um determinado comportamento. Assim, ao ter a clareza de que o sistema de e-learning terá a capacidade de auxiliar o indivíduo por meio de um suporte a realizar suas tarefas com menor esforço durante o processo de aprendizagem, esse passa a ter uma atitude mais favorável ao uso ou a continuidade de uso do sistema. Ou seja, de forma indireta, ou mediada pela facilidade de uso, a presença do suporte técnico influencia de forma positiva $(0,35)$ a percepção de facilidade (H2), que por sua vez influencia, mesmo que de forma limitada, positivamente a atitude (H5). Esse resultado está de acordo com Martins e Kellermanns (2004) ao afirmarem que a disponibilidade de suporte técnico para apoiar o uso de tecnologias se trata de um dos diversos fatores que contribui para a aceitação com maior facilidade da mudança proposta pelo e-learning. Segundo os autores, essa disponibilidade de suporte técnico reduz as preocupações acarretadas pelo processo de mudança ao usuário ao optar por um curso a distância em detrimento do curso presencial da qual está mais habituado. Portando, pode-se argumentar que a presença do suporte técnico aumentam-se as 
chances do aluno continuar a usar o e-learning, reduzindo o índice de evasão.

No que se refere à interatividade, resgata-se $\mathrm{Pi}$ tuch e Lee (2006) que afirmam que a interatividade presente nos processos de aprendizagem faz parte de seus elementos-chave, considerando os indivíduos presentes nestes processos, a saber: alunos e professores, bem como a colaboração que resulta das interações entre estes.

De fato, com a verificação de $\mathrm{H} 1$ - influência positiva da interatividade na utilidade percebida pode-se inferir que aspectos como a comunicação entre os alunos e entre alunos e professores ou tutores proporciona um ambiente de aprendizagem que, de alguma forma, busca simular o ambiente de uma sala de aula presencial, em que se pode esclarecer dúvidas, colaborar ou qualquer outro tipo de participação junto ao professor ou mesmo aos demais "colegas de classe". Os resultados apontaram essa relação como uma das mais fortes do modelo $(0,61)$. Nesse sentido, aumenta-se a percepção de utilidade do curso, bem como a sua intenção de continuar usando o sistema, aumentando, assim, as chances de que o aluno não abandone o curso no meio.

Já em um ambiente de aprendizagem estático, com pouca interação, em que o aluno apenas recebe um conteúdo pronto, esse poderá se sentir desestimulado ao aprendizado na primeira dúvida que surja. Sem formas de entrar em contato com os demais indivíduos que poderiam ajudar na compreensão do conteúdo, poderia ocorrer um desestímulo à continuidade do estudo. Assim, a falta de interatividade, possivelmente, provocará uma dificuldade do usuário em perceber a utilidade do e-learning, acarretando o afastamento dele desse método de ensino, tendo como consequência o não uso e o abandono, conforme argumentou Favero e Franco (2006) em seu trabalho sobre evasão em cursos a distância.

\section{Considerações Finais}

A principal contribuição desde estudo foi avaliar o papel da interatividade e da disponibilidade de suporte técnico na verificação da intenção de continuar a usar um sistema de e-learning por alunos regularmente matriculados nessa modalidade de ensino. Particularmente no que diz respeito ao sistema em si, ou seja, o sistema de e-learning, a interatividade (FAVERO; FRANCO, 2006) e o suporte técnico (ABBAD; ZERBINI; SOUZA, 2010) foram identificados como elementos que poderiam influenciar a intenção de abandonar o curso por parte do aluno. Assim, buscou-se na literatura esses construtos e por meio do modelo TAM foi possível verificar o impacto desses fatores na intenção de uso dos alunos de um curso de graduação a distância de uma Instituição de Ensino Superior. Dessa forma, a pesquisa permitiu concluir que a disponibilidade de suporte técnico e a interatividade são variáveis que influenciam na intenção de continuidade do uso de um sistema de e-learning por parte dos alunos, o que teria como consequência uma redução do abandono do curso, reduzindo, assim, os índices de evasão, confirmando as suposições de Favero e Franco (2006) e Abbad, Zerbini e Souza (2010).

De forma geral, os resultados apontam que a atitude exerce uma influência direta sobre a intenção de uso. Isto significa inferir que quanto mais o aluno tiver uma atitude positiva em relação ao uso do e-learning mais forte será sua intenção de uso do referido sistema. Já facilidade de uso percebida exerce uma influência direta, apesar de limitada, sobre a atitude. Isso significa que a percepção da facilidade de uso do sistema e-learning exerce influência sobre a atitude em relação ao uso por parte dos alunos.

Com relação as variáveis externa introduzidas no modelo, as relações entre a influência da disponibilidade de suporte técnico sobre a facilidade de uso percebida e a influência da interatividade oferecida pelo sistema sobre a percepção utilidade percebida foram ambas confirmadas, comprovando que quanto mais possibilidade haver para o aluno interagir e quando maior for sua percepção de que há um suporte técnico disponível, maior será sua propensão a continuar a usar o sistema de e-learning.

Por fim, do ponto de vista gerencial, a compreensão do impacto desses fatores poderia levar os gestores a adotar medidas para reduzir a desistência do aluno. De acordo com os resultados obtidos, as instituições devem oferecer a seus alunos oportunidades de interação, de modo a atender as necessidades do educando de interagir com colegas e professores e promover o aprendizado de excelência, bem como oferecer disponibilidade de suporte técnico, reduzindo 
sua percepção de dificuldade do processo e, aumentando assim a taxa de conclusão dos alunos matriculados, com consequente diminuição dos índices de evasão.

Há diversos fatores que levam à evasão em $\mathrm{EaD}$, como problemas familiares, dificuldades financeiras, falta de tempo para os estudos (SALES, 2009). Além desses, alguns outros fatores ligados ao sistema podem também ser investigados. Como sugestão para pesquisas futuras, sugere-se verificar a dificuldade de acesso ao sistema devido a problemas técnicos e infraestrutura bem como a qualidade do material disponibilizado via web. Dessa forma, pode-se buscar traçar ações para que o índice de evasão seja reduzido.

\section{REFERÊNCIAS}

ABBAD, G. S.; ZERBINI, T.; SOUZA, D. B. Panorama das pesquisas em educação a distância no Brasil. Estudos de Psicologia, [S.l.], v. 15, n. 3, p. 291298, 2010.

ABED Associação Brasileira de Educação a Distância -. Censo EAD.BR: Relatório Analítico da Aprendizagem a Distância no Brasil 2014. [2014]. Disponível em: < http:// www.abed.org.br/site/pt/midiateca/censo_ead/>. Acesso em: 25 mar. 2016.

ARBAUGH, J. B. Is There an Optimal Design for On-Line MBA Courses? Academy of Management Learning and Education, [S.1.], v. 4, n. 2, p. 135-149, 2005.

ALSHARE, K.A.; ALKHATEEB, F.B. Predicting students usage of Internet emerging economies using an extended technology acceptance model (TAM). Academy of

Educational Leadership Journal, [S.1.], v. 12, n. 2, p. 109-128, 2008.

AJZEN, I. The Theory of Planned Behavior. Organizational Behavior and Human Decision Processes, [S.l.], v. 50, n. 2, p. 179-211, 1991.

CHANG, S.; TUNG, F. An empirical investigation of students' behavioural intentions to use the online learning course websites. British Journal of Educational

Technology, [S.1.], v. 39, n. 1, p. 71-83, 2008.

DAVIS, F. D. Perceived usefulness, perceived ease of use, and user acceptance of information technology. MIS Quarterly, [S.1.], v. 13, n. 3, p. 319-339, 1989.
DAVIS, F. D.; BAGOZZI, R. P.; WARSHAW, P. R. User acceptance of computer technology: a comparison of two theoretical models. Management Science, [S.I.], v. 35, n. 8, p. 982-1003, 1989.

DAVIS, R.; WONG, D. Conceptualizing and Measuring the Optimal Experience of the eLearning Environment. Decision Sciences Journal of Innovative Education, [S.l.], v. 5, n. 1, 2007.

DISHAW, M. T.; STRONG, D. M. Extending the technology acceptance model with task technology fit constructs. Information \& Management, [S.l.], v. 36, p. 9-21, July. 1999.

FAVERO, R.V.; FRANCO, S.R. Um estudo sobre a permanência e a evasão na Educação a Distância, Revista Renote Novas Tecnologias na Educação, [S.l.], v. 4, n, 2, p. 2-10, 2006.

FREITAS, A. S. A implementação do e-learning nas escolas de gestão: um modelo integrado para o processo de alinhamento ambiental. 2009. $330 \mathrm{f}$. Tese (Doutorado em Administração) - Coordenação do Programa de Pós-Graduação em Administração Universidade Católica do Rio de Janeiro, Rio de Janeiro, 2009.

FREITAS, A. S.; BANDEIRA-DE-MELLO, R. Managerial action and sensemaking in e-learning implementation in Brazilian business schools. Computers \& Education, [S.l.], v. 59, n. 4, p. 1.286-1.299, 2012.

HAIR, J. F. et al. Multivariate Data Analysis. 7.ed. Upper Saddle River: Prentice-Hall, 2009.

HU, L.; BENTLER, P. M. Cutoff criteria for fit indexes in covariance structure analysis: conventional criteria versus new alternatives. Structural Equation Modeling, [S.l.], v. 6 , n. 1, p. 1-55, 1999.

HUNG, H.; CHO, V. Continued usage of e-learning communication tools: a study from learner's perspective in Hong Kong. International Journal of Training and Development, [S.l.], v. 3, n. 12, p. 171-187, 2008.

LEE, Y.; KOZAR, K. A.; LARSEN, K. R. T. The technology acceptance model: past, present, and future. Communications of the Association for Information Systems, [S.l.], v. 12, article 50, p. 752- 780, 2003. 
LIU, I. F. et al. Extending the TAM model to explore the factors that affect Intention to Use an Online learning Community. Computers \& Education, [S.1.], v. 54, n. 2, p. 600-610, 2010.

MAIA, M. O uso da tecnologia de informação para a educação a distância no ensino superior. 2003. 294 f. Tese (Doutorado em Administração de Empresas) - FGVEAESP, São Paulo, 2003.

MALHOTRA, N. K. Pesquisa de marketing: uma orientação aplicada. Porto Alegre: Bookman, 2004.

MARTINS, L. L.; KELLERMANNS, F. W. A Model of Business School Students' Acceptance of a Web-Based Course Management System. Academy of Management Learning and Education, [S.1.], v. 3, n. 1, p. 7-26, mar, 2004.

MERCHANT, S. Exploring the influence of cultural values on the acceptance of information technology: an application of the technology acceptance model. Issues. Informing Science and Information Technology, [S.I.], v. 4, p. 431-443, 2007.

NGAI, E. W. T.; POON, J. K. L.; CHAN, Y. H. C. Empirical examination of the adoption on WebCT using TAM. Computers \& Education, [S.l.], v. 48, n .2, p. 250-267, 2007.

ONG, C. S.; LAI, J. Y. Gender differences in perceptions and relationships among dominants of e-learning acceptance. Computers in Human Behavior, [S.I.], v. 22, n. 5, p. 816-829, 2006.

PACHECO, A.; S.; V.; NAKAYAMA, M.; K.; RISSI, M. Evasão e permanência dos estudantes de um curso de administração a distância do sistema universidade aberta do Brasil: uma teoria multiparadigmática. Revista de Ciência da Administração, [S.l.], v. 17, n. 41, p. 65-81, 2015.

PARASURAMAN, A.; GREWAL, D.; KRISHNAN, R. Marketing Research (2nd ed.). South-Western College Pub, 2006.

PARK, N.; LEE, K. M.; CHEONG, P. H. University instructors' acceptance of electronic courseware: An application of the technology acceptance model. Journal of Computer-Mediated Communication, [S.1.], v. 13, n. 1, article 9, 2007.
PARK, S. Y. An Analysis of the Technology Acceptance Model in Understanding University Students' Behavioral Intention to Use e-learning. Educational Technology \&Society, [S.I.], v. 12, n. 3, p. 150-162, 2009.

PAPASTERGIOU, M.; SOLOMONIDOU, C. Gender issues in Internet access and favourite Internet activities among Greek high school pupils inside and outside school.

Computers \& Education, [S.1.], v. 44, n. 4, p. 377-393, 2005.

PITUCH, K. A.; LEE, Y. The influence of system characteristics on e-learning use. Computers \&

Education, [S.1.], v. 47, n. 2, p. 222-244, 2006.

ROCA, J. C.; CHIU, C. M.; MARTÍNEZ, F. J.

Understanding e-learning continuance intention: An extension of the Technology Acceptance Model.

International Journal of Human-Computer Studies, [S.l.], v. 64, n. 8, p. 683-696, 2006.

SAADE, R.; BAHLI, B. The impact of cognitive absorption on perceived usefulness and perceived ease of use in online learning: an extension of the technology acceptance model. Information Management, [S.l.], v. 42, n. 2, p. 317-327, 2005.

SALES, P.A.O. Evasão em cursos a distância: motivos, relacionados às características do curso, do aluno e do contexto de estudo. 2009. 176 f. Dissertação (Mestrado) - Instituto de Psicologia - Universidade de Brasília, UNB. 2009

SILVA, J. S. F. Modelagem de Equações Estruturais - Apresentação de uma Metodologia. 2006. 105 p. Dissertação (Mestrado em Engenharia de Produção) Universidade Federal do Rio Grande do Sul, Porto Alegre, 2006.

STRAUB, D. W. The effect of culture on IT diffusion: e-mail and fax in Japan and the US. Information Systems Research, [S.l.], v. 5, n. 1, p. 23-47, 1994.

VAN RAAIJ, E. M.; SCHEPERS, J. J. L. The Acceptance and Use of a Virtual Learning Environment in China.

Computers \& Education, [S.1.], v. 50, n. 3, p. 838-852, 2008.

VENKATESH, V.; DAVIS, F. D. A theoretical extension of the technology acceptance model:Four longitudinal field studies. Management Science, [S.I.], v. 46, p. 186-204, 2000. 
Angilberto Sabino de Freitas • Jorge Brantes Ferreira • Rodrigo Antônio Garcia • Renata Kurtz

VENKATESH, V. et al. User acceptance of information technology: toward a unified view. MIS Quarterly, [S.l.], v. 27 , n. 3, Sept. p. $425-478,2003$.

VENKATESH, V.; THONG, J.; XU, X. Consumer acceptance and use of information technology: extending the unified theory of acceptance and use of technology.

MIS Quarterly, [S.I.], v. 36, n. 1 p. 157-178, 2012.

VENKATESH, V.; DAVIS, F. D. A theoretical extension of the technology acceptance model: four longitudinal field studies. Management Science, [S.I.], v. 46, n. 2, p. 186204, 2000.

YOUSAFZAI, S.Y.; FOXALL, G. R.; PALLISTER, J. G. Technology Acceptance: a Meta-Analysis of the TAM: Part 1. Journal of Modeling in Management, [S.I.], v. 2, n. 3, p. 251-280, 2007. 